\title{
Enhancement of the density of states at the Fermi level due to oxygen atoms in noble metals
}

\author{
Sudipta Roy Barman ${ }^{1}$ and Aparna Chakrabarti 2,3 \\ ${ }^{1}$ UGC-DAE Consortium for Scientific Research, Khandwa Road, Indore, 452001, Madhya Pradesh, India \\ ${ }^{2}$ Theory and Simulations Laboratory, Raja Ramanna Centre for Advanced Technology, Indore, 452013, India and \\ ${ }^{3}$ Homi Bhabha National Institute, Training School Complex, Anushakti Nagar, Mumbai, 400094, India
}

\begin{abstract}
The interaction of oxygen with noble metals such as silver has been an important topic of research for many decades. Here, we show occurrence of a peak in the density of states (DOS) at the Fermi level $\left(E_{F}\right)$ when oxygen atoms occupy disordered substitutional positions in noble metals such as $\mathrm{Ag}, \mathrm{Au}$ or $\mathrm{Ag}-\mathrm{Au}$ alloy. This results in large enhancement of DOS at $E_{F}$ with respect to $\mathrm{Ag}$ or $\mathrm{Au}$ metal. Its origin is attributed to $\mathrm{O} 2 p$ related disorder broadened flat band that straddles almost all the high symmetry directions of the Brillouin zone. Our work suggests that if a large concentration of disordered oxygen can be realized in nano-structures of noble metals, it may lead to interesting phenomenon.
\end{abstract}

\section{Introduction:}

From way back, the interaction of oxygen with noble metals such as silver has fascinated scientists through different intriguing phenomena such as high solubility of oxygen in liquid $\mathrm{Ag}$ that changes its freezing point[1], dissociation of oxygen molecule at the silver surface[2], incorporation of oxygen atoms (O-atoms) in the bulk[3$6]$ enhancing its catalytic activity $[7,8]$, elongation of free standing silver atom chain by incorporation of alternate O-atoms[9], and anomalous superconducting proximity effect where Ag layer increases the transition temperature $\left(T_{C}\right)$ of $\mathrm{Pb}$ by $15 \%[10]$. Density functional theory (DFT) calculations by Gravil et al.[2] showed that oxygen dissociates into chemisorbed atomic species with no barrier, while Li et al.[8] showed that O-atoms in Ag are responsible for the catalysis. An experimental study by Bao et al.[3] reported that incorporation of O-atoms in the subsurface causes a $3 \%$ expansion of the topmost layer. Eberhart et al. performed a first-principles calculation of the charge density to find that the transport of O-atoms through the octahedral interstitial sites is favored in $\mathrm{Ag}$ and $\mathrm{Cu}$, but not in $\mathrm{Au}[4]$. The authors however did not consider the vacancies, and a later DFT study showed that rather than existing as an $\mathrm{O}$ atom- vacancy pair, the $\mathrm{O}$-atom prefers to occupy the $\mathrm{Ag}$ vacancy i.e. exist at the substitutional site[5]. Baird et al. proposed how an $\mathrm{O}$-atom would diffuse in $\mathrm{Ag}$ by absorbing a phonon to overcome the potential energy barrier[6]. Among the different types of $\mathrm{O}$-atoms that were identified in $\mathrm{Ag}$, $\mathrm{O}_{\beta}$ is the bulk dissolved oxygen, while $\mathrm{O}_{\gamma}$ is a strongly bound species[7]. The stoichiometric oxides of $\mathrm{Ag}$ are semiconducting and these have been studied by DFT primarily to understand why high $T_{C}$ superconductivity is absent in Ag oxides in spite of their similarity with the $\mathrm{Cu}$ oxides[11, 12].

Compared to the bulk, Ag nano-particles (NP) are more susceptible to oxidation[13, 14]. Large concentrations of $\mathrm{O}$ and $\mathrm{C}(0.55$ and 5 times, respectively with respect to $\mathrm{Ag}$ ) in $\mathrm{Ag} @ \mathrm{Au}$ core-shell particles (where $\mathrm{Ag}$ is the core and $\mathrm{Au}$ is the shell) have been reported from $\mathrm{X}-$ ray photoelectron spectroscopy (XPS)[14]. Significantly, the oxygen and carbon signals increase for XPS measurement performed in the bulk sensitive mode, indicating that these are present primarily within the NP and not at the surface. A detailed work using x-ray absorption fine structure spectroscopy and molecular dynamics by Shibata et al. showed existence of vacancies in Au@Ag NP at room temperature that facilitates interface alloying[15]. A scanning tunneling microscopy study showed that with application of electrochemical potential, vacancy clusters can grow and diffuse on $\mathrm{Ag}-\mathrm{Au}$ alloy[16]. This effect is pronounced for small particles of size less than $4.6 \mathrm{~nm}$, where the two metals are nearly randomly distributed. Recently, Yue et al. have demonstrated by transmission electron microscopy and theoretical simulation that oxygen molecules dissociate on the surface of silver NP and diffuse through them to reach the silver/carbon interface and eventually oxidize the carbon[17]. The lattice distortion caused by oxygen concentration gradient within the silver nanoparticles provides the direct evidence for oxygen diffusion.

In this work, we investigate how the electronic structure of fcc noble metals such as Ag and Au is modified by $\mathrm{O}$-atoms in the disordered substitutional (i.e. O-atoms in metal vacancy positions) and interstitial positions using the spin polarized fully relativistic Korringa Kohn Rostoker (SPRKKR) method[18]. For O-atoms in the substitutional position, we find a huge increase in the density of states (DOS) at the Fermi level $\left(E_{F}\right)$ caused by a sharp peak related to $\mathrm{O} 2 p$-like states. The Bloch spectral function, which is the counterpart of dispersion relation for an ordered solid, shows flat and narrow regions straddling the Fermi level along almost all the high symmetry directions of the Brillouin zone (BZ). This is similar to a flat band in an ordered solid that has been broadened by disorder. We show that this phenomenon also occurs in $\mathrm{Au}$, as well as $\mathrm{Ag}-\mathrm{Au}$ alloy. We hence argue that in nano-structures, presence of disordered oxygen bound by dispersive force and stabilized by the noble metal matrix might lead to enhancement of conductivity. 


\section{Methods:}

We have performed self-consistent band structure calculations using SPRKKR method in the atomic sphere approximation[18] within the generalized gradient approximation[19]. The site disorder was treated by coherent potential approximation (CPA) that calculates the configurationally averaged electronic structure self consistently within a mean-field theory. In general, CPA focuses on understanding the scattering of electrons in a material which exhibits spatial inhomogeneity and has turned out to be the most well established way of calculating the influence of disorder on the electronic properties of different materials. The angular momentum expansion up to $l_{\max }=4$ has been used for each atom. The energy convergence criterion and CPA tolerance has been set to $10^{-5}$ Ry. BZ integrations were performed on a $45 \times 45 \times 45$ mesh of $k$-points in the irreducible wedge of the BZ. We have also carried out spin polarized calculations within the SPRKKR formalism. However, we arrive at converged SCF results where the systems turn out to be non-magnetic i.e. the atoms do not carry any partial moment, although the magnetic moments were $0.01 \mu_{B}$ for $\mathrm{Ag}$ and as large as $1 \mu_{B}$ for $\mathrm{O}$ in the starting configuration for all the different $\mathrm{Ag} / \mathrm{Au}-\mathrm{O}$ calculations presented here.

The supercell calculation with full potential linearized augmented plane wave method[20] has been performed with $8000 k$ points in BZ (256 $k$ points in the irreducible BZ). The energy cut-off is about $14.5 \mathrm{Ry}$ and the tolerance for energy convergence is $10^{-4} \mathrm{Ry}$. The cohesive energy $\left(E_{c o h}\right)$, for example for $\mathrm{Ag}_{m} \mathrm{O}_{n}$, has been calculated using $E_{c o h}=E_{t o t}\left(\mathrm{Ag}_{m} \mathrm{O}_{n}\right)-m \times E_{t o t}(\mathrm{Ag})-n \times E_{t o t}(\mathrm{O})$, where $E_{\text {tot }}\left(\mathrm{Ag}_{m} \mathrm{O}_{n}\right), E_{\text {tot }}(\mathrm{Ag})$ and $E_{\text {tot }}(\mathrm{O})$ are the total energies of the concerned $\mathrm{Ag}_{m} \mathrm{O}_{n}$ system, $\mathrm{Ag}$ and $\mathrm{O}$ atoms, respectively. It may be noted that since it is uncertain to which components $\mathrm{Ag}_{m} \mathrm{O}_{n}$ might possibly convert to, its stability against the decomposition into stoichiometric oxide $\left(\mathrm{Ag}_{2} \mathrm{O}, \mathrm{AgO}\right.$, or $\left.\mathrm{Ag}_{2} \mathrm{O}_{3}\right)$ and $\mathrm{O}_{2}$ phases is not addressed in this work.

\section{Results and Discussion:}

The total and partial density of states (PDOS) of $\mathrm{Ag}$ with different amounts of randomly substituted O-atoms are shown in Fig. 1(a-e). The calculations are performed using the equilibrium lattice constant $\left(a_{e q}\right)$ obtained by fitting the variation of the total energy as a function of lattice constant using a polynomial function.

In all cases, $a_{e q}$ turns out to be within a few percent of

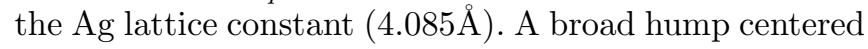
around $-0.7 \mathrm{eV}$ in the total DOS of $\mathrm{Ag}_{88} \mathrm{O}_{12}$ that is absent in $\mathrm{Ag}$ originates from the O $2 p$-like states Fig. 1(a,b). This feature causes an enhancement of the DOS at $E_{F}$ $\left(n_{F}\right)$ by $46 \%$ compared to Ag. The intensity of the hump increases in $\mathrm{Ag}_{75} \mathrm{O}_{25}$, and it shifts very close to $E_{F}(-0.2$ $\mathrm{eV})$. It is striking to note that, for $\mathrm{Ag}_{60} \mathrm{O}_{40}$, this $\mathrm{O} 2 p$ related peak becomes narrower and appears at $E_{F}$, caus-

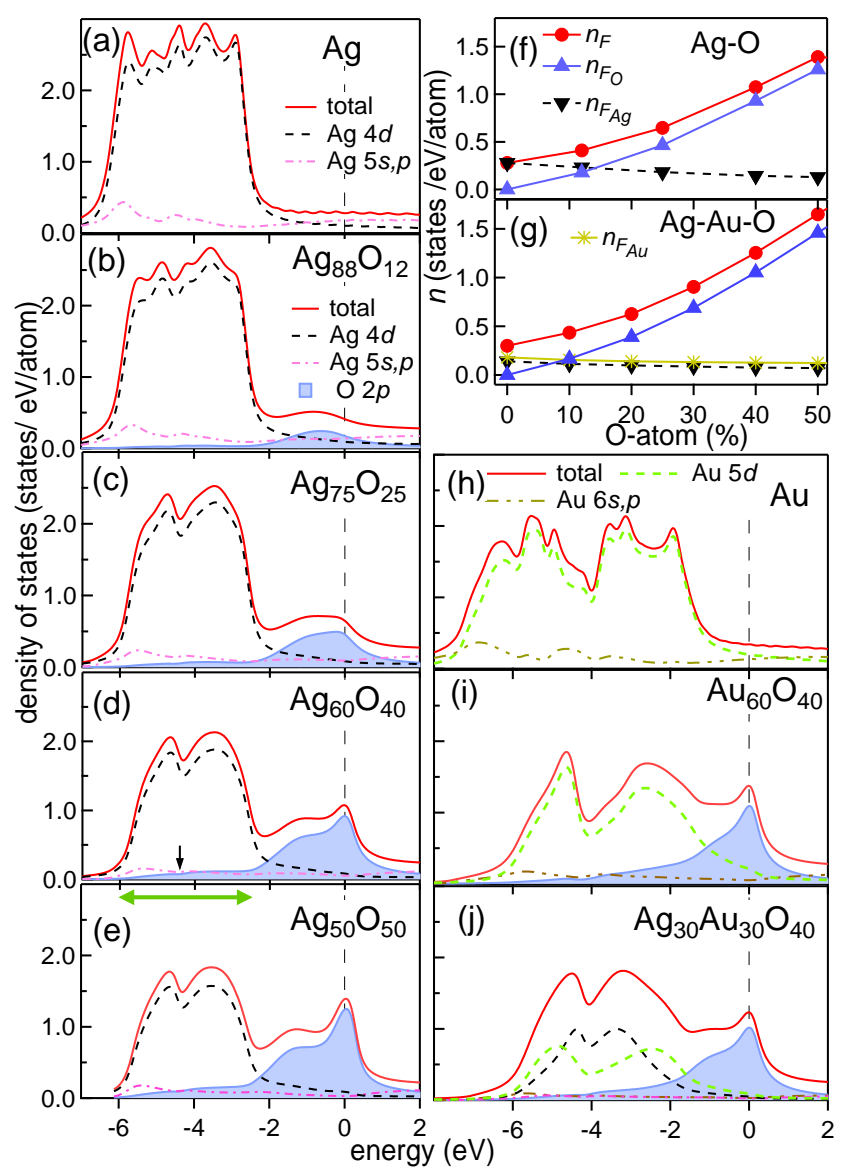

Figure 1: Total and partial density of states of (a) $\mathrm{Ag}$, (b) $\mathrm{Ag}_{88} \mathrm{O}_{12}$, (c) $\mathrm{Ag}_{75} \mathrm{O}_{25}$, (d) $\mathrm{Ag}_{60} \mathrm{O}_{40}$, (e) $\mathrm{Ag}_{50} \mathrm{O}_{50}$, (h) $\mathrm{Au}$, (i) $\mathrm{Au}_{60} \mathrm{O}_{40}$, and $(\mathrm{j}) \mathrm{Ag}_{30} \mathrm{Au}_{30} \mathrm{O}_{40}$. The total DOS at $E_{F}\left(n_{F}\right)$ and the total $\mathrm{Ag}\left(n_{F_{A g}}\right), \mathrm{O}\left(n_{F_{O}}\right)$ and $\mathrm{Au}\left(n_{F_{A u}}\right)$ contributions to $n_{F}$ for (f) $\mathrm{Ag}-\mathrm{O}(\mathrm{g}) \mathrm{Ag}-\mathrm{Au}-\mathrm{O}$, as a function of O-atom content.

ing a very large increase of $n_{F}$ by $286 \%$ (Fig. 1(d,f)). This effect is even more pronounced for $\mathrm{Ag}_{50} \mathrm{O}_{50}$, with a $400 \%$ increase of $n_{F}$, with the maximum of the $\mathrm{O} 2 p$ PDOS peak right at $E_{F}$ (Fig. $\left.1(\mathrm{e}, \mathrm{f})\right)$. The enhancement of $n_{F}$ is also obtained for Au-O (Fig. 1(h,i)), as well as in Ag-Au-O (Fig. 1(g,j)). In fact, for the same oxygen content, $n_{F}$ is largest in Au-O (e.g. $n_{F}=1.37$ states/eV/atom for $\mathrm{Au}_{60} \mathrm{O}_{40}$ in Fig. 1(i)), compared to $n_{F}=1.25$ states/eV/atom for $\mathrm{Au}_{30} \mathrm{Ag}_{30} \mathrm{O}_{40}$ in Fig. 1(j) and $n_{F}=1.07$ states $/ \mathrm{eV} /$ atom for $\mathrm{Ag}_{60} \mathrm{O}_{40}$ in Fig. $\left.1(\mathrm{~d})\right)$. For a disordered system, an equivalent of the band dispersion $E(k)$ can be obtained through the Bloch spectral function, $A_{B}(k, E)$, which is defined as the Fourier transform of the retarded single electron Green's function[21]. For an ordered system, Bloch spectral function (BSF) reduces to a set of $\delta$ functions at the band energies giving $E(k)$. With introduction of disorder, these $\delta$ function peaks are broadened. To find the origin of the DOS peak at $E_{F}$ in Fig. 1, we have calculated the BSF 


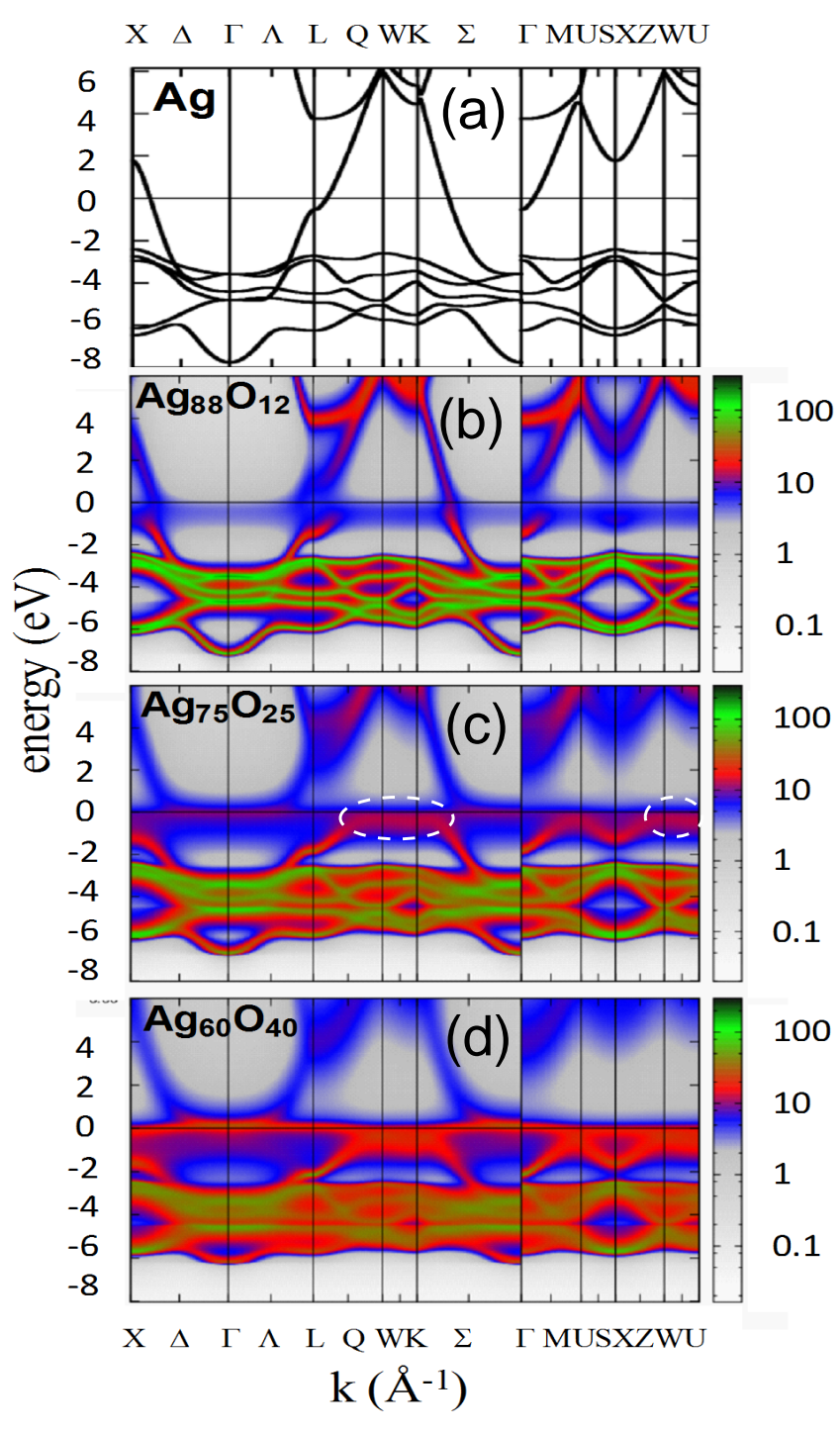

Figure 2: (a) The band dispersion $E(k)$ along the different high symmetry directions of $\mathrm{Ag}$ compared with the Bloch spectral function $A_{B}(k, E)$ along the same directions for (b) $\mathrm{Ag}_{75} \mathrm{O}_{25}$, and (c) $\mathrm{Ag}_{60} \mathrm{O}_{40}$, the color scale on the right side shows the values of $A_{B}(k, E)$ in a.u.

along the high symmetry directions of the fcc BZ. If the BSF of $\mathrm{Ag}_{88} \mathrm{O}_{12}$ in Fig. 2(b) is compared to $E(k)$ of $\mathrm{Ag}$ (Fig. 2(a)), it is observed that the dispersing $\mathrm{Ag} s, p$ band with a kink at the $L$ point splits in the former. The splitting happens because the occupied band shifts down in energy to about $-1.5 \mathrm{eV}$, while the unoccupied part up to $1 \mathrm{eV}$ diminishes in intensity (in Fig. 2(b) see the red colored band around $L$ point, red is about 25 in the relative intensity logarithmic color scale with green as maximum $(\approx 100)$ and white as minimum (zero))). The same happens for the other dispersing Ag $s$ - $p$ bands, e.g. the bands crossing $E_{F}$ at middle of $\Delta-X$ and near $M$ point. Thus, evidently, the contribution of $\mathrm{Ag} s-p$ states to $n_{F}$ decreases. A curious observation from Fig. 2(b) is the appearance of states of low intensity at $E_{F}$ (blue region, 5 in the intensity scale) and we examine how these evolve with larger O-atom content. Strikingly, for $\mathrm{Ag}_{75} \mathrm{O}_{25}$ in Fig. 2(c), parts of the blue region become intense and appear as flat red bands along $W-U$ and $Q-\Sigma$ (both encircled by white dashes). The flat band along $Q-\Sigma$ connects two dispersing bands crossing $L$ and $\Sigma$. A parabolic band emerges with minimum at $X$ at $-1.5 \mathrm{eV}$ and disperses up to the flat band along $W-U$. Weak flat bands (light red color) at $E_{F}$ is also observed along $\Delta-\Gamma-\Lambda$ and $\Sigma-\Gamma$. A spectacular effect occurs for $\mathrm{Ag}_{60} \mathrm{O}_{40}$ (Fig. 2(d)): a flat narrow band (red color) appears at $E_{F}$ spanning all the high symmetry directions from $X-\Gamma-L-W-K-\Gamma$ and $\Gamma-U-X-W-U$ that we have calculated (encircled by yellow dashes). The very wide $k$ range of the flat band, as if it is ubiquitous, along the different directions of the fcc BZ is completely unique. Obviously, this is the origin of the sharp DOS peak at $E_{F}$. The intensity of this flat band further increases for $\mathrm{Ag}_{50} \mathrm{O}_{50}$. The BSF curves of $\mathrm{Au}_{60} \mathrm{O}_{40}$ and $\mathrm{Au}_{30} \mathrm{Ag}_{30} \mathrm{O}_{40}$ also show presence of this intense flat band at $E_{F}$ (Fig.3).

In order to further understand why presence of Oatoms in $\mathrm{Ag}$ or $\mathrm{Au}$ causes such a large enhancement of the DOS at $E_{F}$, we find from the integration of the PDOS up to $E_{F}$ that the total valence charge in O-atom varies from 4.4 to 4.6 i.e. its valency being -0.4 to -0.6 . Thus, the O-atom is almost neutral, with a small excess negative charge $\left(2 p^{4}\right.$ is the valence configuration of oxygen). Its valency is remarkably smaller than the nominal valency of -2 for oxygen, for example, in stoichiometric oxides, where the covalent bonding is predominant and $\mathrm{Ag}$ to $\mathrm{O}$ nearest neighbor $(n n)$ distance is $\approx 2 \AA$ [12]. On the other hand, in $\mathrm{Ag} / \mathrm{Au}-\mathrm{O}$ systems studied here, the $n n$ distance between two $\mathrm{O}$-atoms is substantially larger (e.g. $2.84 \AA$ for $a_{e q}=4.02 \AA$ ) and thus orbital overlap required for covalent bonding is diminished. Rather, it is interesting to note that the van der Waals (vdW) diameter of the O-atom, i.e. the distance between two O-atoms where the minimum of the Lennard-Jones interaction potential occurs, is reported from different estimates to be in the range of $2.8 \AA$ to $3 \AA[22,23]$. Thus, based on the observations that the $\mathrm{O}$-atoms are almost neutral and at a distance equivalent to their $\mathrm{vdW}$ diameter, the dominance of $\mathrm{vdW}$ bonding between $\mathrm{O}$-atoms is expected.

In Fig. 1, the O $2 p$ states extend from $E_{F}$ to $-6 \mathrm{eV}$; it has a shoulder at $-1.3 \mathrm{eV}$ that arises mainly from the $2 p_{x, y}$ states, while the peak at $E_{F}$ has similar contributions from all the three $p$ components. Its noteworthy that in the -2.5 to $-6 \mathrm{eV}$ region (e.g. indicated by green horizontal arrow in Fig. 1(d)), the O $2 p$ PDOS tails out as almost flat with apparently low intensity, but in reality it constitutes about $20 \%$ of the total occupied PDOS. It has a similarity of shape with the $\mathrm{Ag} d$ states, with both exhibiting a minima at $-4.5 \mathrm{eV}$ (black arrow). Such tailing of the $\mathrm{O} 2 p$ PDOS is also observed for $\mathrm{Au}_{60} \mathrm{O}_{40}$ and 


\section{$\mathrm{X} \quad \Delta \quad \Gamma \quad \Lambda \quad \mathrm{L} \quad \mathrm{QW} \quad \mathrm{K} \quad \Sigma \quad \Gamma$ MU SX ZWU}
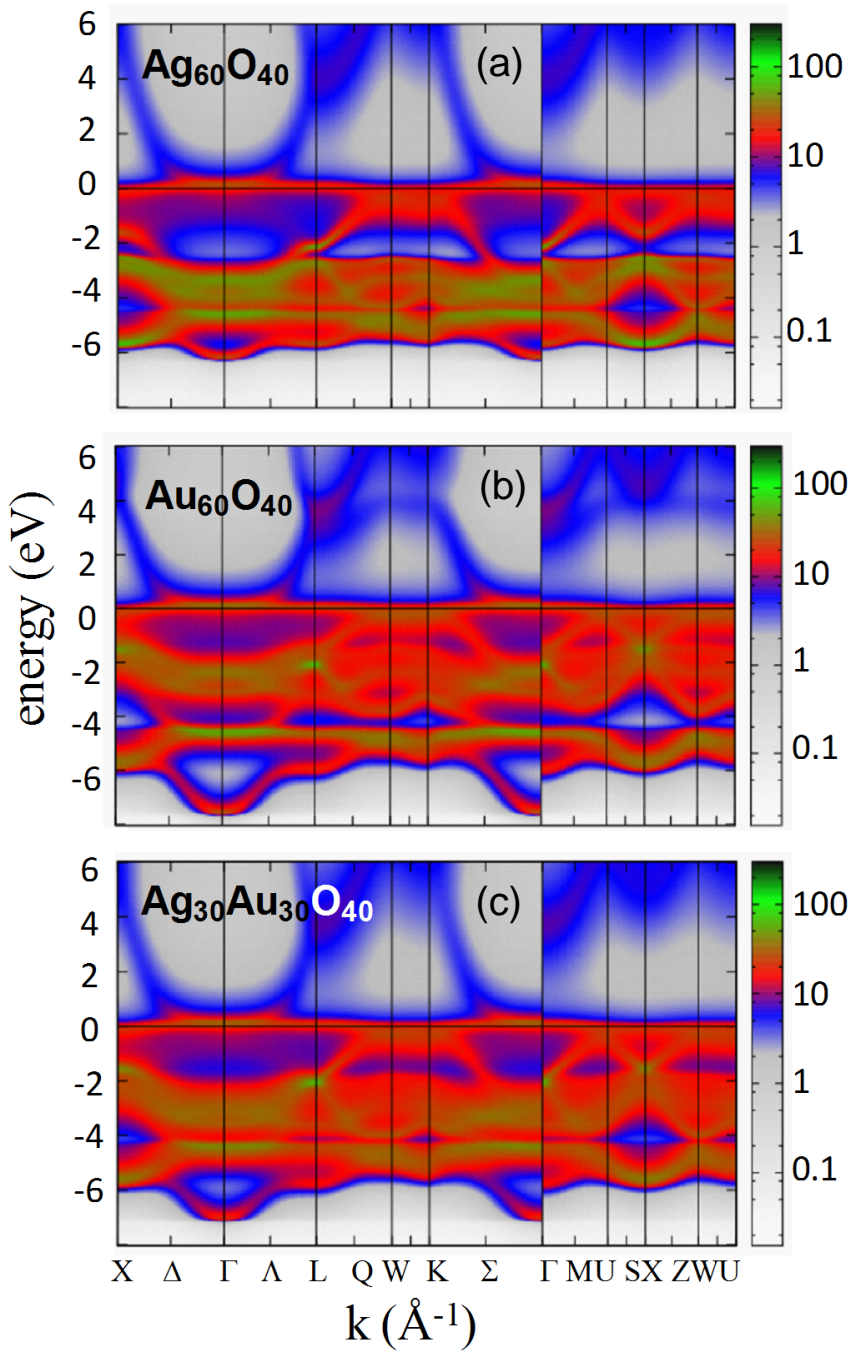

Figure 3: A comparison of the Bloch spectral function $A_{B}(k, E)$ along the different high symmetry directions of the fcc BZ (a) $\mathrm{Ag}_{60} \mathrm{O}_{40}$, (b) $\mathrm{Au}_{60} \mathrm{O}_{40}$, and (c) $\mathrm{Ag}_{30} \mathrm{Au}_{30} \mathrm{O}_{40}$; the color scale on the right side shows the values of $A_{B}(k, E)$ in a.u. A high intensity of $A_{B}(k, E)$ at $E_{F}$ is observed in all cases resembling a disorder broadened flat band.

$\mathrm{Ag}_{30} \mathrm{Au}_{30} \mathrm{O}_{40}$ (Fig. 1(i,j)), indicating hybridization of the O $2 p$ states with the noble metal $d$ states. The cohesive energies $\left(E_{c o h}\right)$ of $\mathrm{Ag}_{60} \mathrm{O}_{40}$ and $\mathrm{Au}_{60} \mathrm{O}_{40}$ have been calculated by considering a $2 \times 2 \times 2$ fcc supercell of 32 atoms with random configurations of 13 O-atoms using full potential linearized augmented plane wave (FPLAPW) method[20]. The DOS shows the O $2 p$ related sharp peak at $E_{F}$ (Fig. 4), which is of similar intensity as in Fig. 1(d). The PDOS also has the hump around -1.3 $\mathrm{eV}$ and the flat DOS region. However, the DOS in this case is highly structured; this is because here we have considered only one particular configuration of O-atoms that is repeated in space in the FPLAPW method and

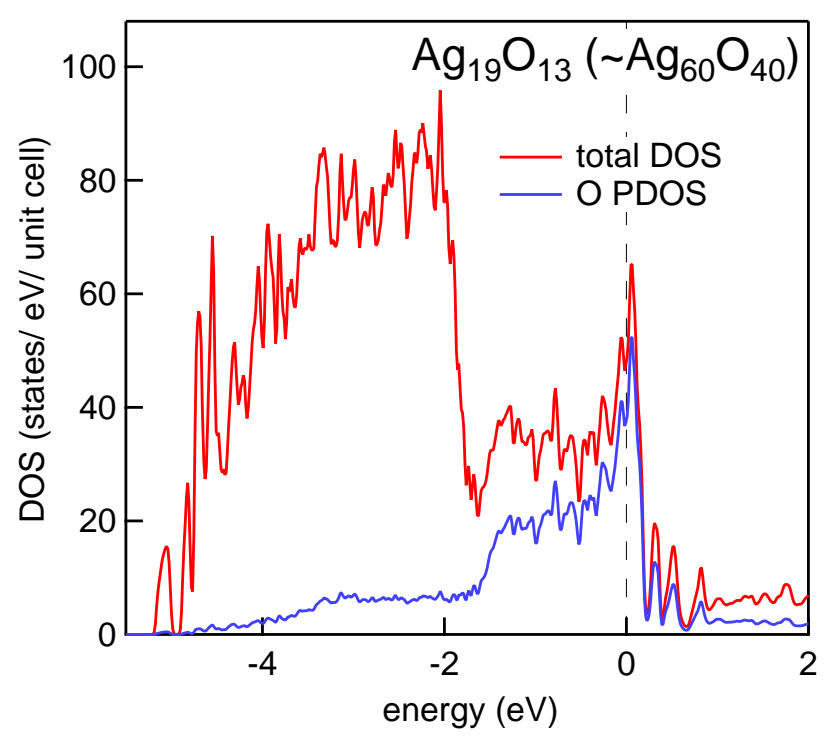

Figure 4: The total and oxygen density of states for $\mathrm{Ag}_{19} \mathrm{O}_{13}$ calculated with a 32 atom fcc supercell using full potential linearized augmented plane wave method.

thus it is not a fully disordered structure. The values of $E_{\text {coh }}$ for $\mathrm{Ag}_{60} \mathrm{O}_{40}$ and $\mathrm{Au}_{60} \mathrm{O}_{40}$ are $2.8 \mathrm{eV} /$ atom and 2.9 $\mathrm{eV} /$ atom, respectively. In comparison, $E_{c o h}$ for $\mathrm{O}$ in fcc structure with same lattice constant is much smaller (1.3 $\mathrm{eV} /$ atom). Thus, hybridization of $\mathrm{O} 2 p$ and the $\mathrm{Ag} / \mathrm{Au}$ $d$ states, as well as their cohesive energies indicate the importance of the noble metal matrix in providing the stability.

We have also considered O-atoms to be randomly distributed in octahedral and tetrahedral interstitial positions of $\mathrm{Ag}$ (Fig. 5(b,c)), since these have been reported in literature as possible sites that oxygen could occupy[46]. In both the interstitial positions, due to the close proximity of $\mathrm{O}$ and $\mathrm{Ag}$ atoms $(2.04 \AA$ for octahedral and $1.77 \AA$ for tetrahedral sites), the Ag $4 d$-O $2 p$ hybridization dominates and the $\mathrm{Ag} 4 d \mathrm{PDOS}$ is largely modified. The $\mathrm{O} 2 p$ PDOS exists over a wide energy range of $10-12$ $\mathrm{eV}$, and does not have any peak at $E_{F}$. Nevertheless, $n_{F}$ increases moderately with O-atom content (Fig. 5(a)). In contrast to $\mathrm{Ag}-\mathrm{O}$ (Fig. 1), this is caused primarily by $\mathrm{Ag} 4 d$ and $5 s, p$ states rather than O $2 p$ states.

A recent work claimed occurrence of room temperature superconductivity in $10 \AA \mathrm{Ag} \mathrm{NP}$ in Au matrix[24], although it must be mentioned at the onset that reproducibility of the data has not been established in the literature yet[25]. It is well-known that high temperature superconductivity could be achieved in materials with large $n_{F}$ and high Debye temperature, however, claim of superconductivity in pure $\mathrm{Ag} @ \mathrm{Au}$ nano-structure (NS) is very curious and even seems unrealistic, since neither $\mathrm{Ag}$ nor $\mathrm{Au}$ are superconducting. In Ref. 24, the au- 


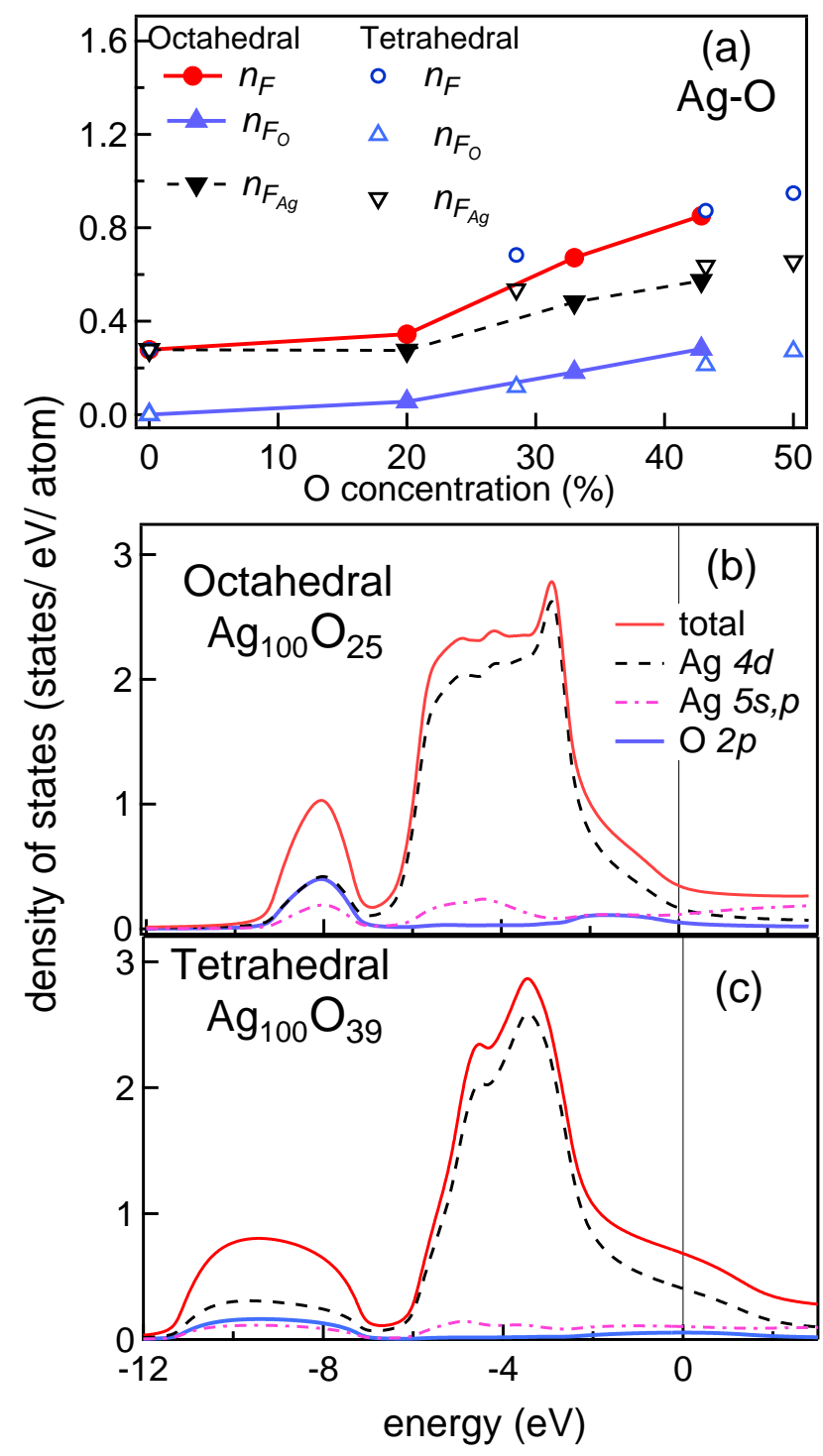

Figure 5: (a) The total DOS at $E_{F}\left(n_{F}\right)$ and the total $\mathrm{Ag}$ $\left(n_{F_{A g}}\right)$ and $\mathrm{O}\left(n_{F_{O}}\right)$ contributions to $n_{F}$ for O-atoms in octahedral and tetrahedral interstitial positions, as a function of O-atom concentration. Total density of states, $\mathrm{Ag} 4 d, \mathrm{Ag} 5 s, p$ and $\mathrm{O} 2 p$ PDOS for (b) octahedral $\mathrm{Ag}_{100} \mathrm{O}_{25}$ and (c) tetrahedral $\mathrm{Ag}_{100} \mathrm{O}_{39}$, where disordered $\mathrm{O}$-atoms and vacancies are considered in the interstitial sites.

thors did not discuss the amount of oxygen present in the $\mathrm{Ag} @ \mathrm{Au}$ NS, although their EDAX data show presence of $\mathrm{C}$ and $\mathrm{O} \mathrm{K} \alpha$ peaks at 0.28 and $0.53 \mathrm{keV}$, respectively. It is well known in literature that $\mathrm{Ag} \mathrm{NP}$ are prone to oxidation $[13,14]$ and the following estimate provides an idea about possible oxygen content: in a $10 \AA \mathrm{Ag} \mathrm{NP}$, there are about 30 atoms. If a single O-atom layer is considered to fully cover this spherical NP, an estimate of the number of O-atoms considering these as hard spheres with atomic diameter of $1 \AA$ will involve 400 O-atoms. The x-ray diffraction pattern shows that the Ag@Au NS

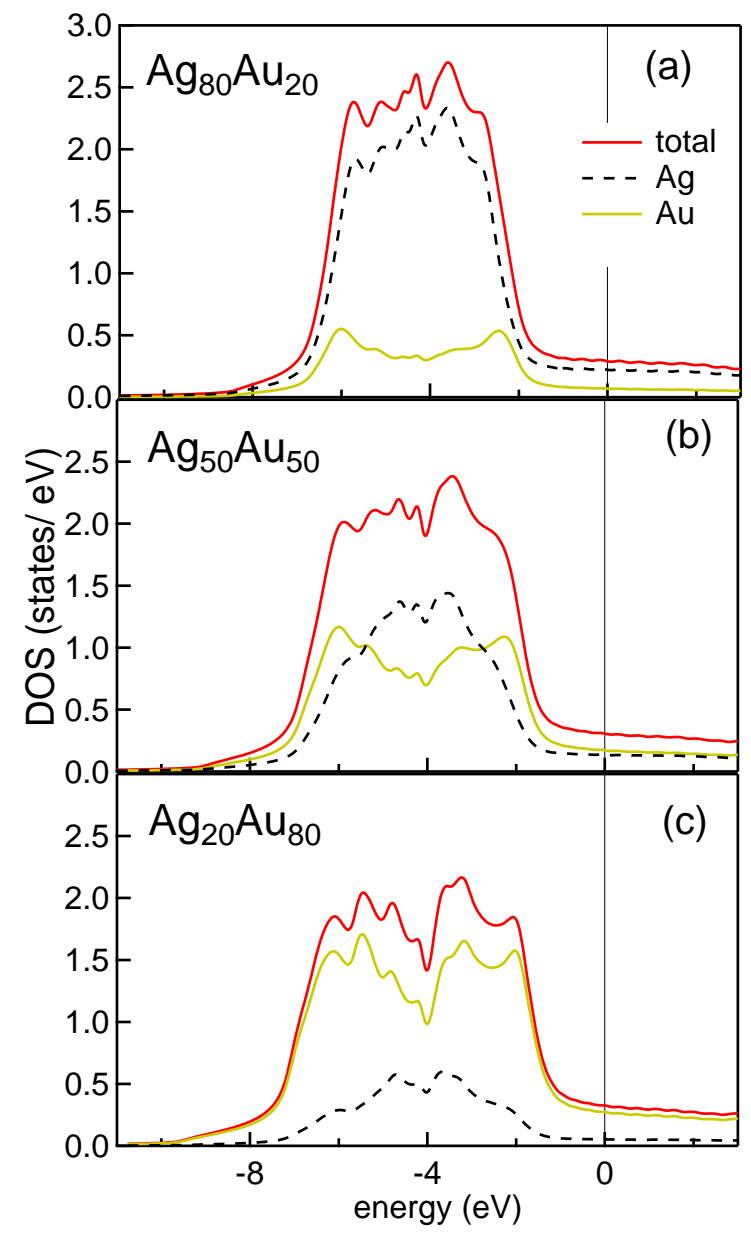

Figure 6: The total, $\mathrm{Ag}$ and Au density of states for (a) $\mathrm{Ag}_{80} \mathrm{Au}_{20}$, (b) $\mathrm{Ag}_{50} \mathrm{Au}_{50}$ and (c) $\mathrm{Ag}_{20} \mathrm{Au}_{80}$. The position of the $\mathrm{Ag} 4 d$ and $\mathrm{Au} 5 d$ states remain essentially unchanged and their relative intensities vary proportionately with composition, with hardly any modification in the DOS of the $s, p$ states at $E_{F}$.

has fcc structure with no extra phases[24]. This shows that the oxygen present in the NS does not form any of the known oxides of $\mathrm{Ag}$ since their structures are not fcc. This indicates that the O-atoms in the NS are present in disordered positions that keep the fcc structure unaffected. We further point out that the O-atoms could be present in the $\mathrm{Ag}$ vacancy (i.e. substitutional) sites from the evidences provided by Shibata et al.[15], Oppenheim et al.[16] and Yue et al.[17], who establish presence of vacancies and O-atoms, their migration through the NP and complete alloying, particularly in small NP of size less than $46 \AA$. Moreover, a DFT study by Crocombette et al. established that it is energetically favored for an oxygenvacancy pair in $\mathrm{Ag}$ to transform to a substitutional O-atom[5]. On the basis of the above arguments, it is conceivable that there is a probability that $\mathrm{O}$-atoms can form local regions of disordered $\mathrm{Ag} / \mathrm{Au}-\mathrm{O}$ with high 


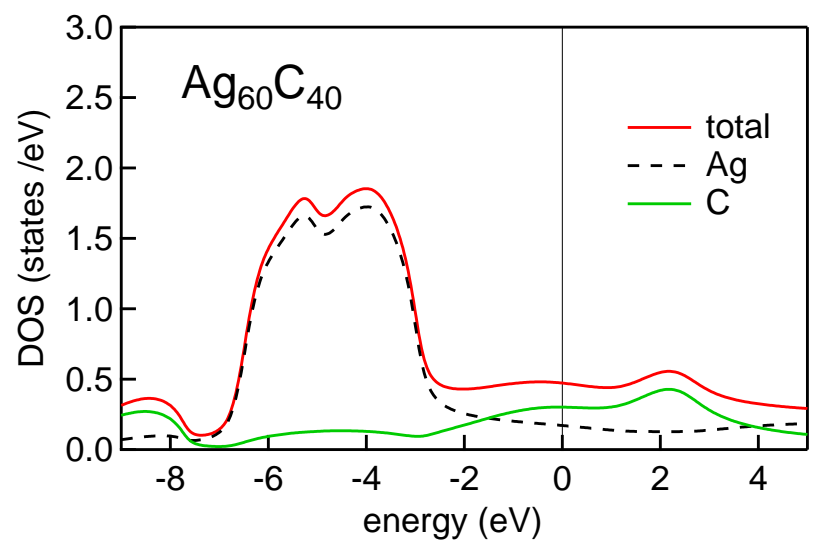

Figure 7: (a) The total, $\mathrm{Ag}$ and $\mathrm{C}$ density of states for $\mathrm{Ag}_{60} \mathrm{C}_{40}$, where $\mathrm{C}$ is in disordered substitutional positions. The calculation has been performed using optimized lattice constant $(3.922 \AA)$. A peak in the $\mathrm{C} 2 p$ PDOS appears above $E_{F}$ at $2.2 \mathrm{eV}$.

oxygen concentration encompassing the $\mathrm{Ag}$ core and $\mathrm{Au}$ matrix, thus possibly forming a connected pathway of regions of high DOS at the Fermi level resulting in high conductivity. While the large DOS at the Fermi level due to occurrence of disordered broadened flat bands as observed in Figs. 1-3 may favor superconductivity[26-28], whether indeed the local oxygen induced superconducting regions may be formed in this kind of systems needs to be probed experimentally.

We also probe the possibility of alloying of $\mathrm{Ag}$ with $\mathrm{Au}$ in an $\mathrm{Ag}-\mathrm{Au}$ alloy. In Fig. 6, we show the DOS of site-disordered $\mathrm{Ag}-\mathrm{Au}$ alloys with composition varying from $\mathrm{Ag}_{80} \mathrm{Au}_{20}$ to $\mathrm{Ag}_{20} \mathrm{Au}_{80}$. Neither any enhancement of $n_{F}$ nor transfer of charge from $\mathrm{Ag}$ to $\mathrm{Au}$ is observed from our SPRKKR calculation. We have also considered disordered carbon in $\mathrm{Ag}(\mathrm{Ag}-\mathrm{C})$, as carbon has been observed to be present in the EDAX signal of $\mathrm{Ag} @ \mathrm{Au}$ NS[24]. The DOS of $\mathrm{Ag}_{60} \mathrm{C}_{40}$ in Fig. 7 calculated using optimized lattice constant of $3.922 \AA$ does not show any peak at $E_{F} . n_{F}$ turns out to be 0.47 states $/ \mathrm{eV} /$ atom, which although is larger than $\mathrm{Ag}$, is considerably less compared to $\mathrm{Ag}_{60} \mathrm{O}_{40}$.

\section{Conclusion:}

We show from density functional theory calculations using SPRKKR method that incorporation of oxygen atoms in disordered substitutional positions of noble metals such as Ag, $\mathrm{Au}$ and $\mathrm{Ag}-\mathrm{Au}$ alloy leads to a large enhancement of the density of states at the Fermi level. The Bloch spectral function, which is the counterpart of dispersion relation for an ordered solid, shows that the peak in the density of states at the Fermi level is related to disorder broadened flat $\mathrm{O} 2 p$ states that straddle almost all the high symmetry directions of the
Brillouin zone. We argue that large concentration of disordered oxygen atoms, if can be realized in noble metal nano-structures, may result in interesting phenomena and stimulate new experiments on noble metal nano-structures.

\section{Acknowledgments:}

We thank the Computer Centre of Raja Ramanna Centre for Advanced Technology, Indore for providing the computational facility. A.C. thanks P.A. Naik for support and encouragement.

[1] E. H. Baker, J. K. Johnstone, Nature 205 (1965) 65-66.

[2] P. A. Gravil, D. M. Bird, J. A. White, Phys. Rev. Lett. 77 (1996) 3933-3936.

[3] X. Bao, J. V. Barth, G. Lehmpfuhl, R. Schuster, Y. Uchida, R. Schlögl, G. Ertl, Surf. Sci. 284 (1993) 14-22.

[4] M. E. Eberhart, M. M. Donovan, R. A. Outlaw, Phys. Rev. B 46 (1992) 12744-12747.

[5] J.-P. Crocombette, H. de Monestrol, F. Willaime, Phys. Rev. B 66 (2002) 024114.

[6] J. K. Baird, T. R. King, C. Stein, J. Phys. Chem. Sol. 60 (1999) 891-894.

[7] A. J. Nagy, G. Mestl, R. Schlögl, J. Catal. 188 (1999) 58-68.

[8] W.-X. Li, C. Stampfl, M. Scheffler Phys. Rev. Lett. 90 (2003) 256102.

[9] W. H. A. Thijssen, D. Marjenburgh, R.H. Bremmer, J.M. van Ruitenbeek, Phys. Rev. Lett. 96 (2006) 026806.

[10] O. Bourgeois, A. Frydman, R.C. Dynes, Phys. Rev. Lett. 88 (2002) 186403.

[11] Y. Quan, W. E. Pickett, Phys. Rev. B 91 (2015) 035121.

[12] J.P. Allen, D.O. Scanlon, and G.W. Watson, Phys. Rev. B 84 (2011) 115141.

[13] C. Shankar, A. T. N. Dao, P. Singh, K. Higashimine, D. M. Mott, S. Maenosono, Nanotech. 23 (2012) 245704.

[14] I. S.- S̆loufová, B. Vlčková, Z. Bastl, T. L. Hasslett, Langmuir 20 (2004) 3407-3415.

[15] T. Shibata, B. A. Bunker, Z. Zhang, D. Meisel, C. F. Vardeman II, J. Daniel Gezelter, J. Am. Chem. Soc. 124 (2002) 11989-11996.

[16] I. C. Oppenheim, D. J. Trevor, C. E. D. Chidsey, P. L. Trevor, K. Sieradzki, Science 254 (1991) 687-689.

[17] Y. Yue, D. Yuchi, P. Guan, J. Xu, L. Guo, J. Liu, Nature Comm. 7 (2016) 12251.

[18] H. Ebert, D. Ködderitzsch, J. Minár, Rep. Prog. Phys. 74 (2011) 096501.

[19] J. P. Perdew, K. Burke, M. Ernzerhof, Phys. Rev. Lett. 77 (1996) 3865-3868.

[20] P. Blaha, K. Schwarz, G. K. H. Madsen, D. Kvasnicka and J. Luitz, WIEN2k, (Karlheinz Schwarz, Techn. Universitat Wien, Austria), 2001. ISBN 3-9501031-1-2.

[21] G. M. Stocks, W. H. Butler, Phys. Rev. Lett. 48 (1982) 55-58; H. Ebert, A. Vernes, J. Banhart, Solid State Comm. 104 (1997) 243-247; H. Ebert, D. Kodderitzsch, J. Minár, Rep. Prog. Phys. 74 (2011) 096501.

[22] L. Pauling, The Nature of the Chemical Bond, Ithaca: Cornell Univ., (1960)

[23] A. Bondi, J. Phys. Chem. 68 (1964) 441. 
[24] D. K. Thapa and A. Pandey, arXiv:1807.08572

[25] B.Skinner, arXiv:1808.02929;

N.Singh, arXiv:1808.10388.

[26] C. C. Tsuei, D. M. Newns, C. C. Chi, P. C. Pattnaik, Phys. Rev. Lett. 65 (1990) 2724-2727.

[27] G. Li, A. Luican, J. M. B. Lopes dos Santos, A. H. Castro
Neto, A. Reina, J. Kong, E. Y. Andrei, Nature Phys. 6 (2010) 109-113.

[28] Y. Cao, V. Fatemi, S. Fang, K. Watanabe, T. Taniguchi, E. Kaxiras, P. J.-Herrero, Nature 556 (2018) 43-50. 